\title{
STRUCTURAL AND ELECTRICAL CONDUCTIVITY STUDIES OF DOPED ZnS
}

\author{
S.V. Sharma and P. Chand* \\ Department of Physics, Indian Institute of Technology, Kanpur-208016, U.P., India
}

(Received April 15, 1992)

\begin{abstract}
The effect of cobalt impurities on transport and structural properties of doped $\mathrm{ZnS}$ has been studied through electrical resistivity, X-ray diffraction and scanning electron microscopy. Correlating the results obtained, it has been shown that the Co concentrations considerably influence the transport properties as well as the structural features, in particular the $\beta \rightarrow \alpha$ phase transformation of $\mathrm{ZnS}$.
\end{abstract}

PACS numbers: $64.70 . \mathrm{Kb}, 64.80 . \mathrm{Gd}, 66.90 .+\mathrm{r}$

\section{Introduction}

Zinc sulphide $(\mathrm{ZnS})$ doped with transition metals and rare-earth ions has been the subject of numerous investigations because of its various practical applications as electroluminescent devices. These devices have a number of attractive advantages such as low power dissipation, capability of large area active light emitting displays, flat cathode ray tubes and flat-panel television [1, 2]. In view of this a great deal of effort has been made towards improving the performance of these devices [1]. It is well-known that ZnS crystallizes in two polymorphic forms, viz. wurtzite $(\alpha-\mathrm{ZnS})$ with hexagonal structure and sphalerite $(\beta-\mathrm{ZnS})$ with cubic structure [3]. It is found that in pure $\mathrm{ZnS}$ the $\beta \rightarrow \alpha$ phase transformation occurs at a temperature around $1297 \mathrm{~K}$ [4]. However, there are some reports which indicate the occurence of this phase transformation at somewhat lower temperature $\approx 1248 \mathrm{~K}$ in the presence of chloride ions [5]. Both the phase transformation rate and the transition temperature of the $\beta \rightarrow \alpha$ phase transformation in $\mathrm{ZnS}$, are found to be greatly affected by the presence of impurities such as $\mathrm{Fe}, \mathrm{Mn}, \mathrm{Cu}$ and chlorine [3]. The role of these impurities in $\beta \rightarrow \alpha$ phase transformation in $\mathrm{ZnS}$ is not yet adequately understood. It would be interesting to see the effect of cobalt chloride impurities since it contains both transition metal and chloride ions to effect the host lattice. In the present investigation the samples of $\mathrm{ZnS}$ doped with

*To whom all correspondence should be addressed. 
cobalt chloride in various molar concentrations have been synthesized and characterized using electron paramagnetic resonance (EPR), electrical resistivity, X-ray diffraction (XRD) and scanning electron microscopy (SEM). The results obtained are reported here.

\section{Experimental}

The synthesis of samples was performed by the usual high temperature solid-state reaction technique from the stoichiometric amounts of high purity $\mathrm{ZnS}$ doped with $\mathrm{Co}^{2+}$ in the molar concentrations varying from 0.01 to $2 \%$. The doped impurity was taken in the form of anhydrous chloride. The powders of above constituents were thoroughly ground and mixed ultrasonically. The mixture was pelletized under the application of a presure of $\approx 8 \mathrm{t} / \mathrm{cm}^{2}$. The pelletized material was then heat treated at a temperature of $1273 \mathrm{~K}$ for 24 hours in argon atmosphere. The samples were labelled according to the dopant concentrations, for example the sample $\mathrm{S} 0.01$ had 0.01 mole $\%$ of $\mathrm{Co}^{2+}$ impurity ions. The other details regarding the sample synthesis are similar to those reported earlier [6]. The resistivity measurements between room temperature (RT) and $1000 \mathrm{~K}$ were performed by the two-probe method. The flat faces of the pellets were silver coated and the samples were sandwiched between two platinum electrodes. The conductivity was evaluated from the average resistance thus measured and the geometry of the pellets. The XRD patterns of the samples were recorded by a Rich-Seifert Isodeflex 2002 diffractometer with $\mathrm{Cu} K_{\alpha}$ radiation. The SEM micrographs were taken using a JEOL 840 JSM Scanning electron microscope. The SEM micrographs of all the samples were taken at the same magnification of 2000 . The samples were checked for paramagnetic impurities with the help of an X-band EPR spectrometer (VARIAN E-109) using $100 \mathrm{kHz}$ phase sensitive detection.

\section{Results and discussion}

The samples were first checked for any EPR signals at RT and liquid nitrogen temperature (LNT). The samples were found to be EPR silent up to LNT. Since a $\mathrm{Co}^{2+}$ ion has fast relaxation time, its EPR signal can only be observed at very low temperatures $(\approx 10 \mathrm{~K})[3]$ which is not accessible in our laboratory at present. EPR silence up to LNT may imply only that no other paramagnetic impurities are present in a detectable concentration in the samples.

Figure 1 shows the plots of electrical conductivity $\sigma$ vs. temperature of doped samples of $\mathrm{ZnS}$. It is evident from the figure that the conductivity of the doped samples increases with the rise in temperature. There is also a change in slope of all the plots at higher temperatures which clearly indicates the presence of two distinct conductivity regions. One of them may be ascribed to extrinsic conductivity region (lower temperature) and the other to the intrinsic conductivity region (higher temperature). This change in slopes occurs at different temperatures for example at about $625 \mathrm{~K}$ for $\mathrm{S} 1$ and $\mathrm{S} 2$ and at about $714 \mathrm{~K}$ for $\mathrm{S} 0.01$ and $\mathrm{S} 0.1$ samples. The activation energies were evaluated from the slopes of these curves and are listed in Table. The activation energy was found to depend on the impurity concentration 


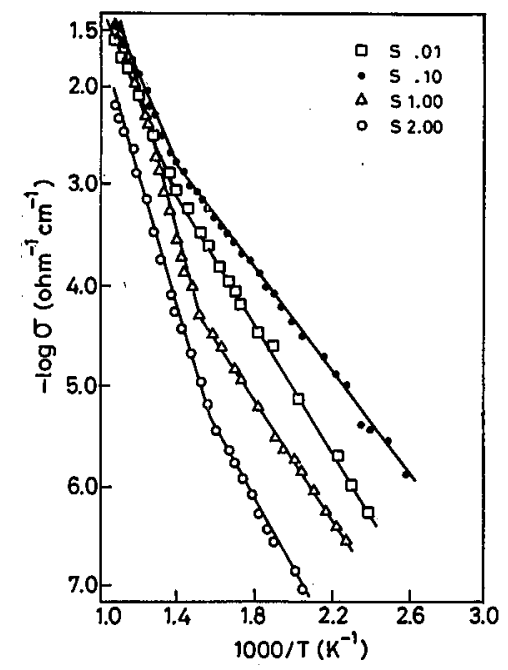

Fig. 1. Temperature dependence of conductivity of the samples S0.01, S0.1, S1 and S2.

TABLE

Important characteristics determined from electrical conductivity, XRD and SEM studies for the $\mathrm{CoCl}_{2}$ doped samples of $\mathrm{ZnS}$.

\begin{tabular}{|c|c|c|c|c|c|}
\hline \multirow{3}{*}{ S. No. } & \multirow{3}{*}{ Samples } & From resistivity & \multicolumn{2}{|c|}{ From XRD } & From SEM \\
\hline & & \multirow{2}{*}{$\begin{array}{c}\text { activation } \\
\text { energy } \\
{[\mathrm{eV}]}\end{array}$} & \multicolumn{2}{|c|}{$\begin{array}{c}\text { percentage of } \\
\text { phase composition }\end{array}$} & \multirow{2}{*}{$\begin{array}{c}\text { average } \\
\text { grain size } \\
{[\mu \mathrm{m}]}\end{array}$} \\
\hline & & & $\alpha-\operatorname{ZnS}$ & $\beta-\mathrm{ZnS}$ & \\
\hline 1 & S0.01 & 0.63 & 38 & 62 & 3 \\
\hline 2 & S0.1 & 0.62 & 46 & 34 & 5 \\
\hline 3 & S1 & 0.51 & 54 & 46 & 8 \\
\hline 4 & S2 & 0.45 & 62 & 38 & 9 \\
\hline
\end{tabular}

in the extrinsic region. From this figure, it has been observed that as the impurity concentration is increased, conductivity first increases (the samples S0.01 and S0.1) and then the trend reverses (the samples S1 and S2). A similar kind of behaviour has been observed in the case of $\mathrm{ZnS}$ doped with $\mathrm{Fe}$ and $\mathrm{Cu}$ [6]. The increase of conductivity with increasing concentration of impurity up to a certain level may be attributed to an increase in the number of charge carriers [7]. At higher concentrations, the mobility of the impurity carriers is expected to decrease hence conductivity also decreases. It is interesting to note that while conductivity data suggests an increase in the number of charge carriers, the samples are EPR silent. This implies that the charge carriers are non-paramagnetic ions or defect centres. 


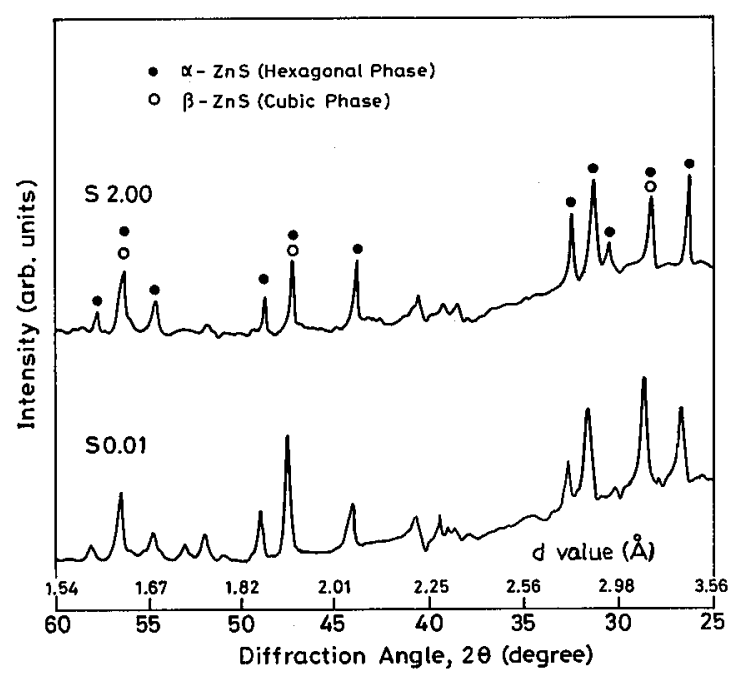

Fig. 2. X-ray diffraction patterns of the samples S0.01 and S2.

The XRD patterns of the samples S0.01 and S2 are shown in Fig. 2. Both the diffractograms of these samples consist of diffraction lines of the hexagonal as well as the cubic phase of $\mathrm{ZnS}$. There are few diffraction lines which correspond to overlapping reflections of hexagonal and cubic phases of $\mathrm{ZnS}$. No peaks corresponding to $\mathrm{CoCl}_{2}$ are detected in any of the samples. This indicates that up to the used concentration levels $\mathrm{CoCl}_{2}$ is soluble in the $\mathrm{ZnS}$ host lattice under the growth conditions used in the present study. The percentages of phase composition of $\alpha$ - and $\beta$ - ZnS have been evaluated from XRD patterns using semiempirical method $[8,9]$ and are given in Table. It may also be noted that as the concentration of doped impurity is increased, the amount of hexagonal phase increases as compared to the cubic phase (Table). This is to be accounted for by the occurrence of $\beta \rightarrow \alpha$ phase transformation of $\mathrm{ZnS}$. This aspect is more clearly evident in the SEM micrographs (Fig. 3). The increase of hexagonal phase with Co concentration may be explained by taking into account the crystallographic difference between the $\alpha$ and $\beta$ polymorphs of $\mathrm{ZnS}$ [3].

The SEM micrographs of the samples S0.01, S0.1, S1 and S2 are shown in Fig. 3. These micrographs show that all the samples contained particles having two different kinds of morphology. One kind of particles has a nearly cubic morphology, while the others have a hexagonal morphology. The relative concentration of the particles with these two morphologies is changing with the doped Co concentration. The samples S0.01 has in majority the cubic type of morphology while the sample S0.1 shows both kinds of morphologies, i.e. cubic and hexagonal. A clear trend however emerges out indicating that in comparable proportions cubic morphology is decreasing and hexagonal morphology is increasing regularly from the samples S0.01 to S2. The sample S2 seems to have hexagonal morphology in majority. The average grain sizes of all the samples have also been evaluated from these 

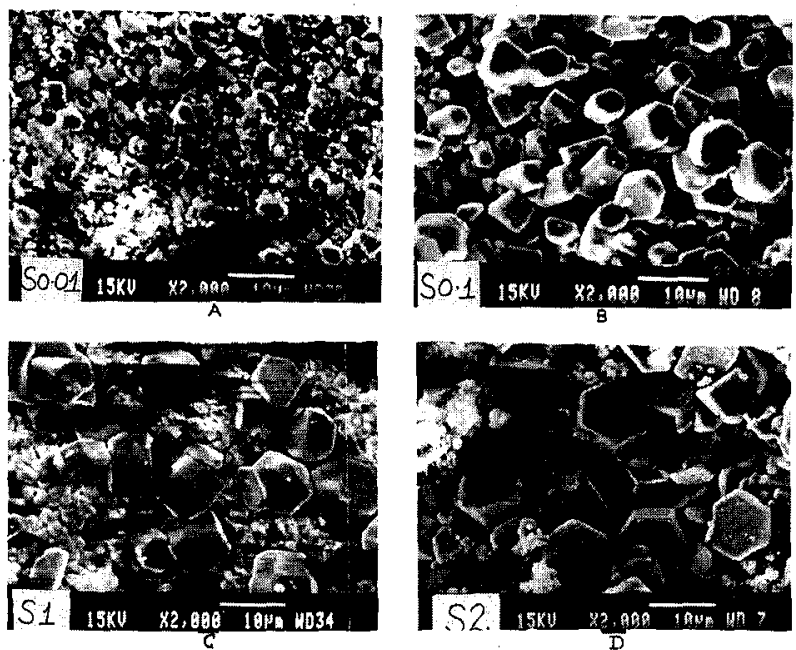

Fig. 3. Scanning electron micrographs of the samples S0.01, S0.1, S1 and S2.

micrographs and are given in Table. This clearly indicates an increase in grain size with the doped Co concentrations which may be correlated with the effect of Co concentration on the $\beta \rightarrow \alpha$ phase transformation of $\mathrm{ZnS}$. This is in conformity with the earlier study [10] which recognized the association of large size grains with hexagonal $(\alpha-\mathrm{ZnS})$ and small size grains with cubic $(\beta-\mathrm{ZnS})$ phase of $\mathrm{ZnS}$-type materials. Thus these observations suggest that $\beta \rightarrow \alpha$ phase transformation is favoured with the presence of $\mathrm{CoCl}_{2}$ impurities in $\mathrm{ZnS}$.

\section{Conclusion}

The electrical conductivity of doped $\mathrm{ZnS}$ has been found to increase with $\mathrm{CoCl}_{2}$ concentrations up to 0.1 mole $\%$ and the conductivity decreases as the concentration of the impurity is further increased. The activation energy has been found to be Co concentration dependent. Further, $\beta \rightarrow \alpha$ phase transformation of $\mathrm{ZnS}$ appears to be favoured by the presence of $\mathrm{CoCl}_{2}$ as revealed by XRD and SEM observations.

\section{References}

[1] K. Okamoto, Y. Hamakawa, Appl. Phys. Lett. 35, 508 (1979).

[2] M.D. Bhise, M. Kaliyadr, A.H. Kital, J. Appl. Phys. 67, 1492 (1990).

[3] M. Aven, J.S. Prener, Physics and Chemistry of II-VI Compounds, North-Holland, Amsterdam 1967.

[4] D.W. Balletyne, J. Phys. Chem. Solids 10, 242 (1959).

[5] A. Kremhella, Sylvania Technologist 8, 11 (1955).

[6] S.V. Sharma, R.J. Singh, Bull. Mater. Sci. 14, 71 (1991). 
[7] C. Kittel, Introduction to Solid State Physics, 4th ed., Wiley Eastern Private, New Delhi 1974.

[8] B.D. Cullity, Elements of X-ray Diffraction, Addison Wesley, London 1959.

[9] P.R. Bote, P.K. Patil, J.K. Nandgave, D.R.L. Pawar, Solid State Commun. 79, 5 (1991).

[10] Y. Ma, R.H. Bube, J. Electrochem. Soc. (USA) 124, 1430 (1977). 